\title{
Negative Regulation of Cell-proliferation of Adult Stem Cells in the Midgut and Hindgut by dMats in Drosophila melanogaster
}

\author{
Yan Yan (Corresponding author) \\ Cell and Developmental Biology Program, the Pennsylvania State University \\ University Park, Pennsylvania 16802, USA \\ Tel: 717-343-2307 E-mail: yanyanms@gmail.com \\ Bin Kang \\ Department of Biology, University of Pennsylvania, Pennsylvania 19104, USA
}

Received: March 21, 2011 Accepted: April 7, 2011 doi:10.5539/ijb.v3n3p105

\begin{abstract}
The existence of adult stem cells in the midgut and hindgut of Drosophila has been reported. The components of Hippo pathway such as dWarts and dYki were involved in the regulation of stem cell proliferation in the midgut. In this study, we reported the role of dMats in the control of stem cell proliferation in the midgut and hindgut of adult fruit fly. The eGFP signals driven by dmats indicated that dMats expressed in small diploid cells in the adult midgut and hindgut. By inducing MARCM cell clones, the dmats -/-cell clones in the midgut exhibited a bigger cell number than wild type cell clones and tissue outgrowth. Similarly, the dmats-/- mutation also caused more cell numbers in hindgut. Thus, dMats was critical to control stem cell proliferation in midgut as well as in hindgut. Our results implicated an important regulatory role for Hippo pathway in the regulation of adult stem cell proliferation.
\end{abstract}

Keywords: dMats, Adult stem cell, Midgut, Hindgut

\section{Introduction}

In the adult stage of organism, many types of tissues are wore out or damaged due to physiological or pathological situation, thus new cells need to be generated to replenish the lost tissues. Those cells that possess the ability to renew through mitotic dividing and the ability to differentiate into several different types of cells are adult stem cells. The stem cells existing in the adult stage of organisms can be further divided into somatic stem cells (i.e., skeletal muscle stem cells, epithelial stem cells, CNS neural stem cells) and germline stem cells. The epithelial stem cells are located on the organ surface or intestine lumen surface (Blanpain et al. 2007; Raff, 2003; Fuchs et al. 2004). Epithelial tissues, providing protection for organs, are responsible for the epithelial tissue tumor (carcinoma), the highest incidence tumor among other tissue cancers.

It has been reported that intestinal stem cells (ISC) are located in the midgut and hindgut, which are subjected to be regulated by multiple pathways (Ohlstein et al. 2006; Micchelli et al. 2006; Ohlstein et al.2007; Takashima et al. 2008). The ISC is the only type cell that proliferated in the adult midgut (Ohlstein et al. 2006; Micchelli et al. 2006; Staley et al. 2010). The regulatory mechanism of stem cells in midgut is different from that in hindgut: midgut does not form a niche structure around its ISC, while hindgut forms niches in the surroundings of its ISC. Being multipotent, one intestinal stem cell in midgut can generate one progenitor cell (EB), two differentiated cells: enterocytes (EC) and enteroendocrine cells (ee). The ISC can perform self-renewal through an asymmetric cell division to generate one enteroblast (EB) cell and one ISC cell (Ohlstein et al. 2006; Micchelli et al. 2006). The regulation of ISC self-renewal and cell fate determination of descendant cells is controlled through Notch-Delta pathway. If an ISC expresses high level of cytoplasmic Delta, Notch pathway will be activated and the cytoplasmic Delta in its daughter cell will be down regulated, then the daughter cell will become enterocytes (EC); while if an ISC expresses low vesicular Delta or has impaired Notch pathway signaling, the daughter cell will become enteroendocrine cells (Ohlstein et al. 2007).

The hindgut epithelia tissue is mainly composited by large cuboidal epithelial cells that can secrete cuticle (Takashima et al. 2008). ISC was identified in the anterior narrow segment of hindgut, named as hindgut 
proliferation zone (HPZ). The self-renewal of hindgut ISCs and their descendant's proliferation and differentiation were controlled by locally emanating Wingless $(\mathrm{Wg})$ and Hedgehog (Hh) pathways. The limited expression of $\mathrm{Wg}$ in the spindle cell zone (SCZ) of HPZ maintains self-renewal of ISCs in a slow cycling as a niche signals. The $\mathrm{Wg}$ signals are low in the round cell zone (RCZ), where cells perform rapid proliferation. In RCZ, Hh signals controlled these cells proliferation and the onset of differentiation (Takashima et al.2008).

Hippo pathway was identified as a tumor suppressor pathway since loss-of-function mutants of Hippo pathway components exhibited overgrowth phenotypes and inhibited apoptosis, suggesting this pathway plays a key role in inhibiting cell proliferation and promoting apoptosis during Drosophila development (Saucedo et al. 2007; Harvey et al. 2007; Hariharan et al. 2006; Xu et al. 1995 ; Wu et al. 2003; Huang et al. 2005; Pan, 2005; Ho et al.2010). It is known that other Hippo pathway components regulated midgut stem cell. For example, it was demonstrated that dWarts and dYki played a role in the transition from low to high-level ISC proliferation to facilitate regeneration of midgut tissue when damage occurred. Activation of dYki by midgut damage, bacterial infection or activation of JNK pathway induced a nonautonomous increase in ISC proliferation (Staley et al. 2010; Shaw et al. 2010; Ren et al. 2010; Karpowicz et al. 2010). dMats, one of the key components, is a scaffold protein activating dWarts kinase activity, which in turn phosphorylats transcription co-activation dYki to inhibit cell number increase (Lai et al. 2005; Wei et al. 2007; Shimizu et al. 2008). However, it is largely unknown if the dMats could regulate stem cell in midgut. Moreover, it is complete unknown if dMats could be involved in the regulation of the hindgut stem cell either.

Therefore it is highly demanded and possible to test if dMats is involved in controlling stem cell proliferation in both midgut and hindgut. In this study, the role of dMats in the ISC proliferation was examined and our evidence showed that loss-of-function of dMats increased cell proliferation of ISC residing in both midgut and hindgut.

\section{Material and methods}

\subsection{Fly Strains used in this study.}

Drosophila strains used in this study were listed in the Table 1.

\subsection{Drosophila strains culture}

All strain stocks were grown in $10 \mathrm{ml}$ disposable vials in a $25 \mathrm{C}$ incubator unless different temperature was indicated. Hard agar fly food was made according to the recipe (Wirtz et al. 1982). After heat shock, strains were kept in the $18-20^{\circ} \mathrm{C}$ to reduce the background signals.

\subsection{Drosophila dissection and microscopes}

Adult flies were anesthetized with $\mathrm{CO}_{2}$. Dissection was performed under Olympus dissecting microscope with 1x PBS $\left(0.08 \% \mathrm{NaCl}, 0.002 \% \mathrm{KCl}, 0.0115 \% \mathrm{Na}_{2} \mathrm{HPO}_{4} \cdot 7 \mathrm{H}_{2} \mathrm{O}, 0.002 \% \mathrm{KH}_{2} \mathrm{PO}_{4}\right)$. Isolated fly guts were fixed

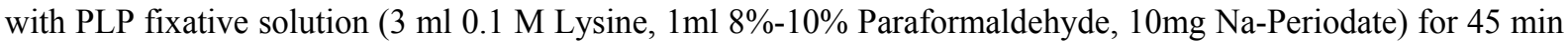
at $4^{\circ} \mathrm{C}$ in the dark. Samples were washed with PBS 5-10 min for 3 times and then counter stained with 5-10 uM Draq5 (Biostatus Limited, UK) for 5-30 min or 300nM DAPI (Sigma, MO) for 1-5 min. The samples were observed and pictures were taken with Olympus Flowview 1000 Confocal Laser Scanning Microscope and Olympus BX60 fluorescence microscope.

\subsection{Expression of Drosophila genomic Mats (dgMats) driving nuclear GFP in fruit fly}

To check the expression of dmats in fly tissues, dmats driving eGFP strain was made (Fig.1A) (Yang et al. 2008). Wild-type genomic mats (the genomic Mats (dgMats) including regulatory element/enhancer of dmats, covering the 2236-bp region of the D. melanogaster mats gene), were amplified by PCR, and inserted into the KpnI and $X b a$ I sites of pBluescript II SK+ vector. Positive constructs were selected and verified by DNA sequencing. The primers used were mats-FW, 5'-CGGGGTACCGATTGCG AAATGTTAGGGAC-3' and mats-RV, 5'-CGCGGTCTAGAGTGTCAATACACGATAAACC-3'. The mats gene genomic fragments were cut with $K p n \mathrm{I}$ and $X b a \mathrm{I}$, and subcloned into the KpnI and NheI sites of pH-Stinger transformation vector (Barolo et al. 2000) that contains nuclear-targeted GFP under the control of a minimal promoter (XbaI and NheI are isocaudomers with the same GATC extensions). The fly strain ( $w$; $p[g M a t s] e G F P$ ) containing the pH-Stringer dgmats-eGFP construct was made by microinjection according to the standard protocols (Rubin and Spradling, 1982). The minimum promoter in $\mathrm{pH}$ Stinger only exhibited very low GFP signals, which was treated as background (personal communication with Prof Lai Zhi-chun) (Yang et al. 2008)

2.5 Generation of GFP labeled Mosaic Analysis with a Repressible Cell Marker (MARCM) clones to analyze the cell proliferation of stem cells by heat shock in fruit fly

The process of MARCM is summarized in many references (Pan, 2007). 82 MARCM ( $w$; hsFlp, UAS-GFP; FRT82B, Tub-GAL4, Tub-Gal80/TM6B) crossed with $w$; FRT82B mats ${ }^{\text {roo }} /$ TM6B or $w$, FRT82B mats ${ }^{\text {e235 }} /$ TM6B 
and $y, w ; P[r y+;$ hsneo]; FRT82B P[y+ry+]96E. The female progenies $(w ; h s F l p, U A S-G F P$; FRT82B, Tub-GAL4, Tub-Gal80/mats ${ }^{\text {roo }}$ or w; hsFlp, UAS-GFP; FRT82B, Tub-GAL4, Tub-Gal80/ mats ${ }^{\text {e235 }}$ and $w$; hsFlp, UAS-GFP; FRT82B, Tub-GAL4, Tub-Gal80/96E) were collected. Heat shock was given at $37^{\circ} \mathrm{C}$ for one hour when flies were 2-7days after enclosed. GFP signals were observed under confocal microscope.

\section{Results}

3.1 dMats expression was found in the posterior part of midgut of adult Drosophila melanogaster where adult stem cell resides

Although it is known that dMats functioned in inhibiting cell proliferation and promoting apoptosis in many tissues of imaginal discs (Lai et al. 2005; Shimizu et al. 2008; Ho et al. 2010), whether dMats played a role in the cell division at the adult stage, such as adult stem cell division is unknown. In order to clarify the functions of dMats in the adult fly, we initially examined its expression in the adult fly gut, since the adult stem cells were found to reside in the epithelial tissue of gut (Ohlstein et al. 2006; Micchelli et al. 2006; Ohlstein et al. 2007; Takashima et al., 2008). dmats driving eGFP strain (w; p[gmats]eGFP) was used to study the expression of dMats. Under light microscope, the structure of adult midgut of Drosophila includes cardia (anterior), megan, 1st loop, 2nd loop, posterior midgut, and pylorus (Sup.1) (Micchelli et al. 2006). The dMats GFP signals were found in megan, two loops and posterior part of adult midgut with low intensity. The most often region where dMats expression was observed was the posterior part of midgut (11 positive samples $/ 12$ total samples) while the least expression was in anterior part (0/12) of midgut (Fig.1A, Table 2). The GFP signals were found only in the guts obtained from the strain transformed by dMats driving eGFP vector (Fig. 1D). Similarly, it was reported that the adult stem cells were identified mainly in the posterior part of midgut (Ohlstein et al. 2006; Micchelli et al. 2006; Ohlstein et al. 2007), thus we focused our attention in this region.

The ISC cells of fruit flies are located at the basal location of gut tissue and contained diploid content of DNA. The daughter cells thus were generated and pushed to the lumen of the gut when ISC performs cell proliferation (Micchelli et al. 2006). The GFP signals could be also seen at the basal location of the midgut where ISC normally resides (Fig.1C and 1E), implying that dMats might express in stem cells of adult midgut.

\section{2 dMats signals were cell type specifically expressed in the midgut}

There are several types of cells in adult midgut: ISC, enteroblast (EB), enterocytes (EC) and enteroendocrine (ee). The ISC, EB and ee cells are diploid cells (2n) while EC cells are polyploid cells (8n) (Ohlstein et al. 2006; Micchelli et al. 2006). In our nuclear staining pictures, we found both diploid cells and polypoid cells were in the midgut (Fig.1G). By merging the signals of GFP and the nuclear staining, we found that the GFP signals mainly co-localized with small nucleus (2n) (Fig.1H, Sup.2). Similar as reported, the ISC cell was observed close to its two daughter cells that were all diploid cells (Ohlstein et al. 2006). In this study, three diploid nuclei expressing dMats GFP were observed to line up together; according to their location, one of them might be the ISC (Sup.3) (Ohlstein et al. 2006). The dMats GFP signals in the three diploid cells were different. So the dMats expression level might be an indicator of ISC cell, and its daughter cells.

\subsection{Loss-of-function of dmats-/- mutant cell clones exhibited enhanced cell proliferation in the midgut}

We identified that dMats most likely expressed in the ISC of midgut by the dMats driving GFP signals. However, we have not known if dMats played functions in the ISC of midgut. Thus the MARCM cell clones were induced in the fruit fly guts, by which the dividing cells in midgut were labeled by GFP. It is known that dividing cells are stem cells in adult midgut (Ohlstein et al. 2006; Micchelli et al. 2006; Staley et al. 2010), so by this method, we can determine if loss-of-function dMats could affect ISC proliferation in adult midgut. If the dmats-/- cell clones do not show any difference from wild type clones in cell numbers, the dMats most likely does not regulate ISC proliferation in midgut. But if the dmats-/- cell clones do show more cell numbers than the wild type clones, then the dMats not only does express in the ISC but also regulates ISC cell proliferation. From this experiment, the cell numbers between wild type clones and dmats-/- cell clones were significantly different during the same time (Fig 2). It was clear that the dmats-/- cell clones contained more cells than the wild type clones at all the time points when samples were harvested. The wild type clones contained small numbers of cells ( $1.9 \pm 0.34$ cells/clone) in about 8 days (Fig.2C, $2 \mathrm{E}$ ); while the dmats mutant clones contained $4.72 \pm 0.72$ cells/clone (Fig.2D, 2E). The difference of cell numbers in two types of MARCM clones was statistically significant $(\mathrm{P}<0.01)$ (Fig.2E). Therefore, loss-of-function dMats in the midgut enhanced the ISC cell proliferation than wild type, which supported the hypothesis that dMats played a role in ISC cell proliferation in midgut.

\subsection{Loss-of-function of dmats-/- mutant cell clones showed tissue outgrowth in the posterior part of midgut}

As we know, loss-of-function of dmats caused cell over-proliferation and tumor growth in fruit fly (Lai et al.2005). In this study, we also observed that loss-of-function of dmats in the adult midgut caused similar tissue 
outgrowth (Fig.3D, 3F). Since the stem cell is the only dividing cells in adult midgut (Fox et al. 2009), MARCM clones in midgut showed that dMats played a role in stem cell proliferation. At the $8^{\text {th }}$ day after MARCM clone induction, the dmats-/- mutant cell clones in midgut were bigger than wild type cell clones (Fig.2C, 2D). The tissue outgrowth and enlarged posterior part of midgut could be found on the $15^{\text {th }}$ day and $35^{\text {th }}$ day after MARCM clone generation, which was identified in several independent cases in the mutant sample group; however, such tissue outgrowth did not find in the wild type samples (Fig.3). The tissue outgrowth in the dmats-/- clones suggested that dMats is very important in controlling the ISC proliferation in adult midgut.

\section{5 dMats expression was identified in the hindgut with low level}

The hindgut of fruit fly was defined from the pylorus to the rectum, which functions as large intestine (Takashima et al.2008; Fox et al. 2009) (Fig.1B). In the hindgut of adult fruit fly, the stem cell study is a little controversy. Hindgut stem cells are reported to reside in the anterior part called hindgut proliferation zone (HPZ), where small diploid cells are found to perform cell proliferation during the adult stage for the tissue renewal; however, it is also reported that the stem cell in hindgut are not constitute active but stress-induced active (Takashima et al. 2008; Fox et al. 2009) In this study, using strains of $w, p[g M a t] e G F P$, dMats GFP signals were found in the HPZ (6 out of 8 samples) with low level (Fig.1A, 1C, Table 2). It also was found the dMats GFP signals with a little higher level in the posterior part (ileum) where the differentiated cells are located (Fig.1C, Table 2). These results were consistent with the previously work that dMats expression can be identified in the adult tissues (Yang et al. 2008).

\subsection{In hindgut, loss-of-function of dmats resulted in enlarged cell clones and tissue outgrowth}

After heat shock, we found that wild type gut was less often to generate MARCM clones (about 1-2 in about 10-20 samples) than mutant guts (in almost all samples can be seen MARCM clone generated), which is consistent with the previously report that normally the activity of ISC in hindgut is very low (Fox et al. 2009). Besides, the wild type clones (dmats $+/+$ ) contained only $0.67 \pm 0.26$ cells/clone on the $35^{\text {th }}$ day after treatment; however, the mutant clones (dmats-/-) showed bigger cell numbers $(2.267 \pm 0.42$ cells/clone) in the hindgut (Fig.2E, Fig.4). The cell numbers' difference between dmats-/- clones and wild type clone was significant $(\mathrm{p}<0.01$, Student's t-Test) (Fig.2E). Some mutant clones also showed the tumor like tissue outgrowth on the 35th day post heat shock treatment, which also changed the morphology of the hindgut (Fig 4C, 4D). In both wild type gut and mutant gut, the stem cell clones in HPZ (Fig.4A, 4E) and transient clones in the differentiated hindgut were all observed in this experiment (Fig.4B, 4C, 4D) (Takashima et al. 2008), which was consistent with the distribution of dMats signals in the hindgut.

\section{Discussion}

dMats is a negative regulator in cell proliferation during fruit fly development: loss-of-function of dmats caused outgrowth of several tissues and organs such as head, notum, compound eyes and neurons ( Lai et al. 2005; Shimizu et al. 2008; Ho et al. 2010). However, the expression and functions of dMats are not clear in the adult fruit fly. In the adult stage of organism, there are many occasions where tissues need to generate new cells to supply the loss of tissues such as epithelial tissue, germline cells and blood cells etc (Blanpain et al., 2007; Raff 2003; Fuchs et al., 2004). The epithelia tissues of midgut and hindgut of fruit flies were reported to be actively replaced during the living time of flies (Ohlstein et al. 2006; Micchelli et al.2006; Ohlstein et al. 2007; Takashima et al.2008). As one type of somatic stem cell, epithelial stem cell is very important in the stem cell research since epithelial tissue protects the organs and it is replenished during all the life span of any organisms. Dysfunction of the self-renewal of epithelial stem cell may cause tissue loss or cancers (Blanpain et al. 2007; Raff 2003; Fuchs et al. 2004). ISC cells in the adult fruit fly were identified and several significant developmental pathways were involved in its regulatory mechanisms such as Notch, Wnt, Hh etc (Ohlstein et al. 2006; Micchelli et al. 2006; Ohlstein et al. 2007; Takashima et al., 2008). The components of hippo pathway such as Warts and Yorkie were also found in the ISC cells of midgut (Staley et al. 2010; Shaw et al. 2010; Ren et al. 2010; Karpowicz et al., 2010). The ISC cells in midgut and hindgut are all diploid small cells, which reside at the basal location of the tissue and usually are adjacent to the daughter cells. The ISC cells perform self-renew and one of their daughter cells becomes differentiated. In midgut, the differentiated cells are enterocytes (EC) and enteroendocrine cells (ee) (Ohlstein et al. 2006; Micchelli et al. 2006; Ohlstein et al. 2007). While in hindgut, ISC cells resided in HPZ which includes spindle cell zone (SCZ), round cell zone (RCZ) and differentiated hindgut cells (hgd), ISC cells generate the large cuboidal epithelial cells (Takashima et al., 2008).

In this study, the functions of dmats in the ISC cell proliferation were examined in the midgut and hindgut. Consistent with previously work, dmats signals were found to exist from Mega to the posterior part of adult midgut and hindgut (Table2) (Yang et al, 2008). Among these locations, the dMats signals were the most frequent at the posterior part of midgut than any other parts of midgut (Fig.1A, Table 2), which is coincident 
with other lab's work about ISC in midgut (Ohlstein et al. 2006; Micchelli et al. 2006; Ohlstein et al. 2007). It was also observed that there ISC and their daughters (small diploid cells) lined together as other lab's report (Sup2). The dMats signals showed different levels in these cells (Sup2). The cell producing low dMats signals and residing in the basal location of midgut tissue showed more intense nuclear staining than the other two diploid cells, indicating this cell was under active dividing. Thus, this cell would be possible to be the ISC cell. It would be not surprising that ISC cell expresses dMats in a low level due to dMats's inhibition of cell proliferation (Lai et al., 2005; Wei et al, 2007; Shimizu et al., 2008). Based on the function of dMats in imaginal discs of fruit fly, it is highly likely that the two cells with higher dMats signals were daughter cells of ISC (Fig.2, Sup.2, 3). Therefore, our data implied that dMats might express in the midgut ISC cells and some differentiated cells.

The induced MARCM clones in midgut further suggested that dMats regulated the ISC cells existing in the posterior part of midgut. The dividing cells in the midgut forming MARCM clones are ISCs (Ohlstein et al. 2006; Micchelli et al. 2006; Staley et al. 2010). The dmats mutant clones increased their cell numbers faster than wild type clones indicating the dmats function was required for the ISC cell proliferation (Fig.2E). Loss-of-function of dmats in ISC caused tissue outgrowth and tumor like structure in the posterior part of midgut suggesting that dMats inhibited ISC cell proliferation in such a way that the ISC could perform cell proliferation at a low rate/slow pace according to the environment clues such as EGFR ligands or lesions (Fig.3) (Staley et al. 2010; Shaw et al. 2010; Ren et al.2010; Karpowicz et al. 2010).

Interestingly, to our knowledge, the results of hindgut are the first evidence until now that dMats also functions in the ISC cells of hindgut. In the hindgut, dMats signals were observed in the HPZ where the ISC cells were located and in the posterior part of hindgut as well (Takashima et al., 2008). The signals of dMats in the HPZ region were very low but significant comparing to the other parts of hindgut such as differentiated hindgut (Fig.3). Thus, dMats might play roles in the ISC and other cells in the hindgut. Though the wild type hindgut was induced MARCM clones in very low frequency as reported (Fox et al. 2009), suggesting that ISC in wild type hindgut was less active. However, it seems not a GFP leaking expression since the untreated samples were not observed similar GFP clones. In the induced MARCM clones, the dmats mutant clones were obviously bigger than the wild type clones. dmats mutant clones grew to big tissue clog and changed the morphology of the hindgut (Fig.4). These observations demonstrated that loss-of-function of dmats in ISC cell impaired the ISC division control and would result in tumor in hindgut.

The distribution of dMats expression implied that dMats not only regulated ISC cells but also regulated differentiated cells in midgut and hindgut. When the function of dmats was impaired, ISC cells might overgrow without limitation and became tumors in the midgut and hindgut. It was found that dMats expressed in some cells in high level in the other parts of midgut such as loops and megan, but loss-of-function of dmats in these cells only brought a bigger cell clones and did not cause tissue outgrowth and tumor like structures (Fig.1, Fig.2). Similarly, dMats signals were identified in the posterior hindgut some differentiated cells with higher level, where the dmats mutant clones also bigger than the wild type clones (Fig.2, Fig.4). When the function of dmats was lost in the differentiated cells, cell clones became bigger in the hindgut, which suggested that there are other signals functioning in the differentiated cells proliferation.

In summary, this study on the dmats function in cell proliferation of ISC in the adult midgut and hindgut provided initial evidence. The expression of dMats was found in the adult stage of midgut and hindgut. Similar to its function in the imaginal discs development, loss-of-function of dMats caused MARCM cell clones over-proliferation in midgut and hindgut. Therefore this study implied the ISC cells in midgut and hindgut required the function of dMats for proliferation control.

\section{Acknowledgements}

The authors thank Prof Lai Zhi-Chun (Penn State University) for his support. Authors also thanks Prof. Xu Tian (Yale University), Prof. Norbert Perrimon (Harvard University), and Prof. Kenneth D Irvine (University of Rutgers), Prof. Georg Halder and Prof. Andreas Bergmann (University of Texas) for providing fly strains in this study. Authors also thank the technique support from the Cytometry Facility and Electron Microscopy Facility of PSU (University Park). This work is supported by NSF funding (0641914) awarded to Dr. Lai Zhi-Chun.

\section{References}

Barolo S, Carver LA \& Posakony JW. (2000). GFP and $\beta$-galactosidase transformation vectors for promoter/enhancer analysis in Drosophila, BioTechniques, 29,726-232.

Blanpain C, Horsley V \& Fuchs E. (2007). Epithelial stem cells: turning over new leaves. Cell, 128, 445-458. 
Fox DT \& Spradling AC. (2009). The Drosophila hindgut lacks constitutively active adult stem cells but proliferates in response to tissue damage. Cell Stem Cell, 5, 290-297.

Fuchs E, Tumbar T \& Guasch G. (2004). Socializing with the neighbors: stem cells and their niche. Cell, 116, 769-778.

Harvey K \& Tapon N. (2007). The Salvador-Warts-Hippo pathway - an emerging tumour-suppressor network. Nat Rev Cancer, 7, 182-191.

Hariharan IK \& Bilder D. (2006). Regulation of imaginal disc growth by tumor-suppressor genes in Drosophila. Annu Rev Genet, 40, 335-361.

Ho LL, Wei X, Shimizu T \& Lai Z. (2010). Mob as tumor suppressor is activated at the cell membrane to control tissue growth and organ size in Drosophila. Dev Biol, 337, 274-283.

Huang J, Wu S, Barrera J, Matthews K \& Pan D. (2005). The Hippo Signaling Pathway Coordinately Regulates Cell Proliferation and Apoptosis by Inactivating Yorkie, the Drosophila Homolog of YAP. Cell, 122, 421-434.

Karpowicz P, Perez J \& Perrimon N. (2010). The Hippo tumor suppressor pathway regulates intestinal stem cell regeneration. Development, 137, 4135-4145.

Lai ZC, Wei X, Shimizu T, Ramos E, Rohrbaugh M, Nikolaidis N, Ho LL \& Li Y. (2005). Control of Cell Proliferation and Apoptosis by Mob as Tumor Suppressor, Mats. Cell,120, 675-685.

Micchelli CA \& Perrimon N. (2006). Evidence that stem cells reside in the adult Drosophila midgut epithelium. Nature, 439, 475-479.

Ohlstein B \& Spradling A. (2006). The adult Drosophila posterior midgut is maintained by pluripotent stem cells. Nature, 439, 470-474.

Ohlstein B \& Spradling A. (2007). Multipotent Drosophila intestinal stem cells specify daughter cell fates by differential notch signaling. Science, 315, 988-992.

Pan DJ (2007). Hippo signaling in organ size control. Genes \& Dev, 21, 886-897.

Raff M. (2003). Adult stem cell plasticity: fact or artifact? Annu Rev Cell Dev Biol, 19, 1-22.

Ren F, Wang B, Yue T, Yun EY, Ip YT \& Jiang J. (2010). Hippo signaling regulates Drosophila intestine stem cell proliferation through multiple pathways. Proc Natl Acad Sci USA,107, 21064-21069.

Rubin GM \& Spradling AC. (1982). Genetic transformation of Drosophila with transposable element vectors. Science, 218, 348-353.

Saucedo LJ \& Edgar BA. (2007). Filling out the Hippo pathway. Nat Rev Mol Cell Biol, 8, 613-621.

Shaw RL, Kohlmaier A, Polesello C, Veelken C, Edgar BA \& Tapon N. (2010). The Hippo pathway regulates intestinal stem cell proliferation during Drosophila adult midgut regeneration. Development, 137, 4147-4158.

Shimizu T, Ho LL \& Lai ZC. (2008). The mob as tumor suppressor Gene Is Essential for Early Development and Regulates Tissue Growth in Drosophila. Genetics, 178, 957-965.

Staley BK \& Irvine KD (2010). Warts and Yorkie mediate intestinal regeneration by influencing stem cell proliferation. Curr Biol, 20, 1580-1587.

Takashima S, Mkrtchyan M, Younossi-Hartenstein A, Merriam JR \& Hartenstein V. (2008) The behaviour of Drosophila adult hindgut stem cells is controlled by Wnt and Hh signalling. Nature, 454, 651-655.

Tao W, Zhang S, Turenchalk GS, Stewart RA, St John MA, Chen W \& Xu T. (1999). Human homologue of the Drosophila melanogaster lats tumour suppressor modulates CDC2 activity. Nat Genet, 21(2): 177-181.

Wei X, Shimizu T \& Lai ZC. (2007). Mob as tumor suppressor is activated by Hippo kinase for growth inhibition in Drosophila. The EMBO Journal, 26, 1772-1781.

Wirtz RA \& Semey HG. (1982). The Drosophila kitchen-equipment, media preparation and supplies. Dros. Inf. Serv, 58, 176-180.

Wu S, Huang J, Dong J \& Pan D. (2003). hippo Encodes a Ste-20 Family Protein Kinase that Restricts Cell Proliferation and Promotes Apoptosis in Conjunction with salvador and warts. Cell, 114, 445- 456.

Xu T,Wang W,Zhang S, Stewart RA \& Yu W. (1995). Identifying tumor suppressors in genetic mosaics: the Drosophila lats gene encodes a putative protein kinase. Development, 121, 1053-1063. 
Yang Y, Gupta V, Ho LL, Zhou B, Fan Q, Zhu Z, Zhang W\& Lai ZC. (2008). Both upstream and downstream intergenic regions are critical for the mob as tumor suppressor gene activity in Drosophila. FEBS Lett, 582, 1766-1770.

Table 1. Drosophila strains used in this study

\begin{tabular}{|c|c|c|}
\hline & Genotype & \\
\hline 1 & $w^{1118}$ & (Lai et al., 2005) \\
\hline 2 & $w, p[g M a t] e G F P$ & (Lai et al., 2005) \\
\hline 3 & $w ; F R T 82 B$ mats $^{e 235} / T M 6 B$ & (Lai et al., 2005) \\
\hline 4 & w; FRT82B mats $^{\text {roo }} /$ TM6B & (Lai et al., 2005) \\
\hline 5 & $y, w ;$ FRT82B lats $^{x I} / T M 6 B$ & (Tao et al., 1999) \\
\hline 6 & $y, w ; P[r y+;$ hsneo $] ;$ FRT82B P $[y+r y+] 96 E$ & (Tao et al., 1999) \\
\hline 7 & y,w,UAS-GFP, hsflp; tubulin-Gal4;FRT82B, tub-Gal80/TM6B & (Micchelli et al. 2006) \\
\hline 8 & $y, w, h s f l p ; F R T 82 B, h s p i-M y c$ & (Micchelli et al. 2006) \\
\hline
\end{tabular}

Table 2. Expression distribution of eGFP through dMat promoter in adult Drosophila. ${ }^{1}$

\begin{tabular}{|c|c|c|c|}
\hline \multirow{3}{*}{ midgut } & location & [g mats]-eGFP & mock (wild type) \\
\cline { 2 - 4 } & cardia & $0 / 12$ & $0 / 11$ \\
\cline { 2 - 4 } & megan & $1 / 12$ & $0 / 11$ \\
\cline { 2 - 4 } & $1^{\text {st }}$ loop & $4 / 12$ & $0 / 11$ \\
\cline { 2 - 4 } & $2^{\text {nd }}$ loop & $2 / 12$ & $0 / 11$ \\
\cline { 2 - 4 } & Posterior midgut (pylorus) & $11 / 12$ & $0 / 11$ \\
\hline \multirow{3}{*}{ hindgut } & HPZ & $6 / 8$ & $0 / 10$ \\
\cline { 2 - 4 } & Differentiated hindgut & $5 / 8$ & $0 / 10$ \\
\hline
\end{tabular}

1 The fly strain is $w, p[g M a t] e G F P$.

2 HPZ: hindgut proliferation zone 

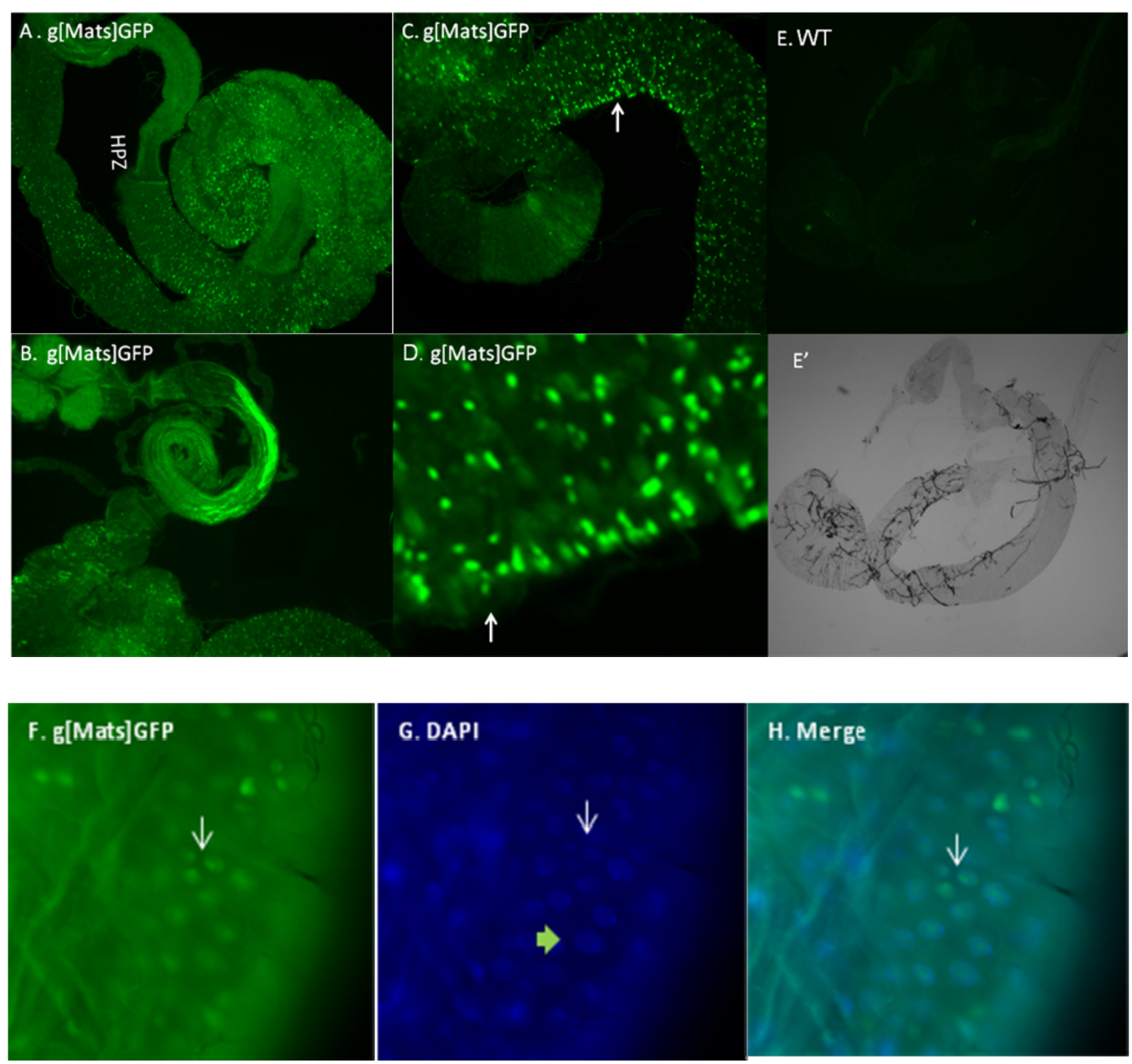

Figure 1. GFP signals showed dMats expression in the midgut and hindgut of adult fruit fly

Fly guts of strains ( $w, p[g M a t] e G F P$ ) containing the dmats driving eGFP signals were observed and the picture showed the nuclear GFP distribution in the midgut and hindgut. A, The GFP signals of dMats showed in the most part of midgut and the hindgut( HPZ with low but clear GFP signals), 100x. B, The GFP dMats signals showed the cells expressing dMats were located in the posterior of midgut and in the hindgut, 200x. C, The GFP signals showed the cells expressing dMats were located at the basal location of the midgut (arrow), 200x. D, the higher magnification of Fig 1C showed GFP signals in the cells of the basal location. E and E', the negative samples without dmats GFP signals in the midgut and hindgut, 40x. F, the GFP signals showed the dMats expressed in small diploid cells of midgut (arrow). G, the picture showed the cell nuclear staining with DAPI in the tissue of the midgut; among them, the small nuclei are diploid cells (2n)(white arrow) and these bigger are polyploidy cells (8n)(green arrow). H, the merged view of DAPI and GFP, 400x. 

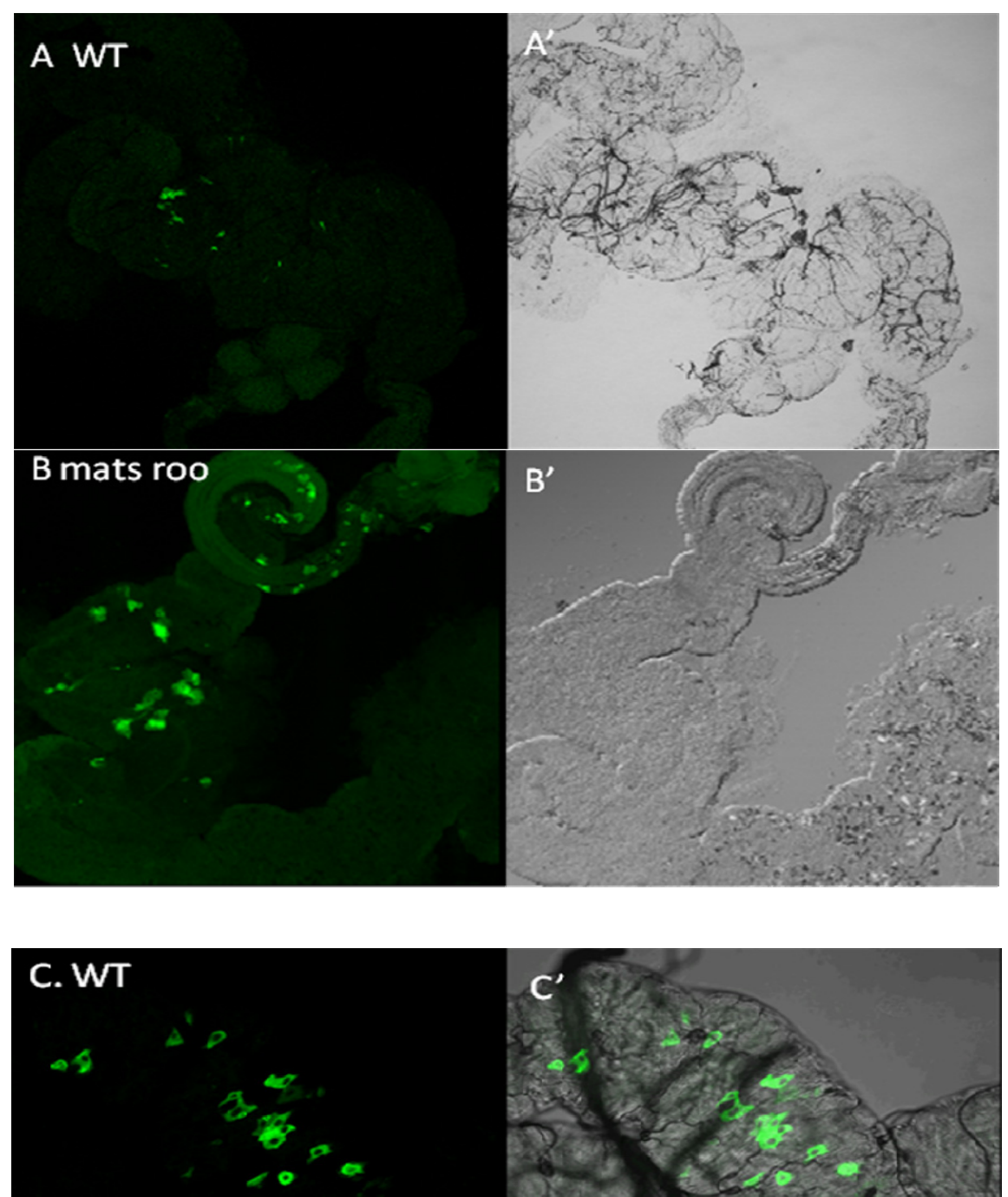

\section{D. mats roo}

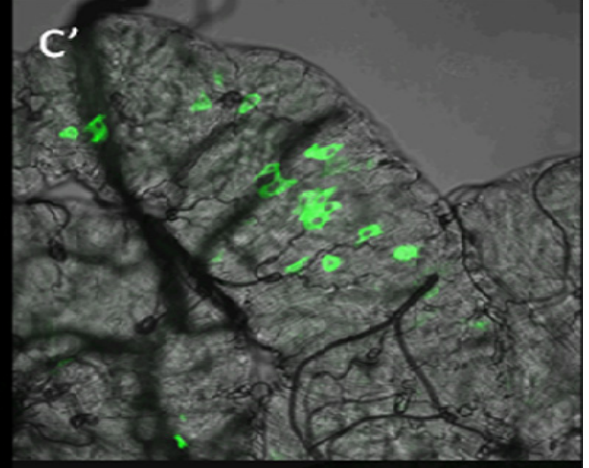

$D^{\prime}$

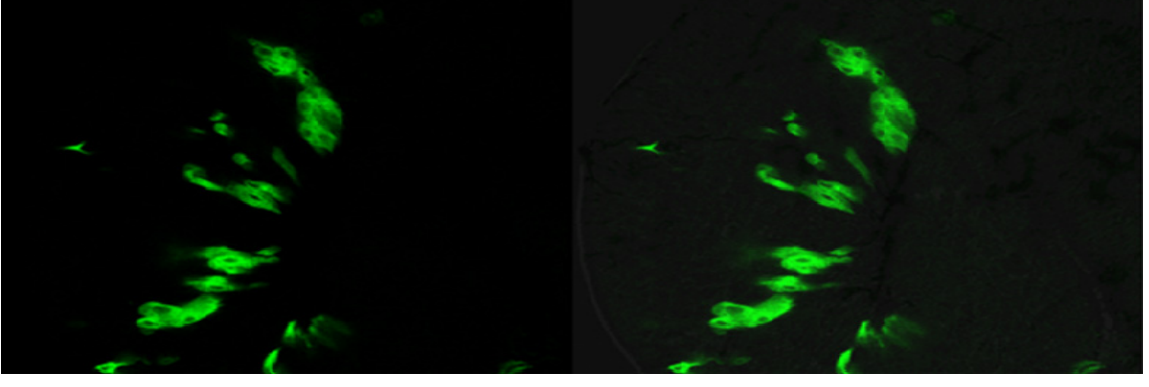




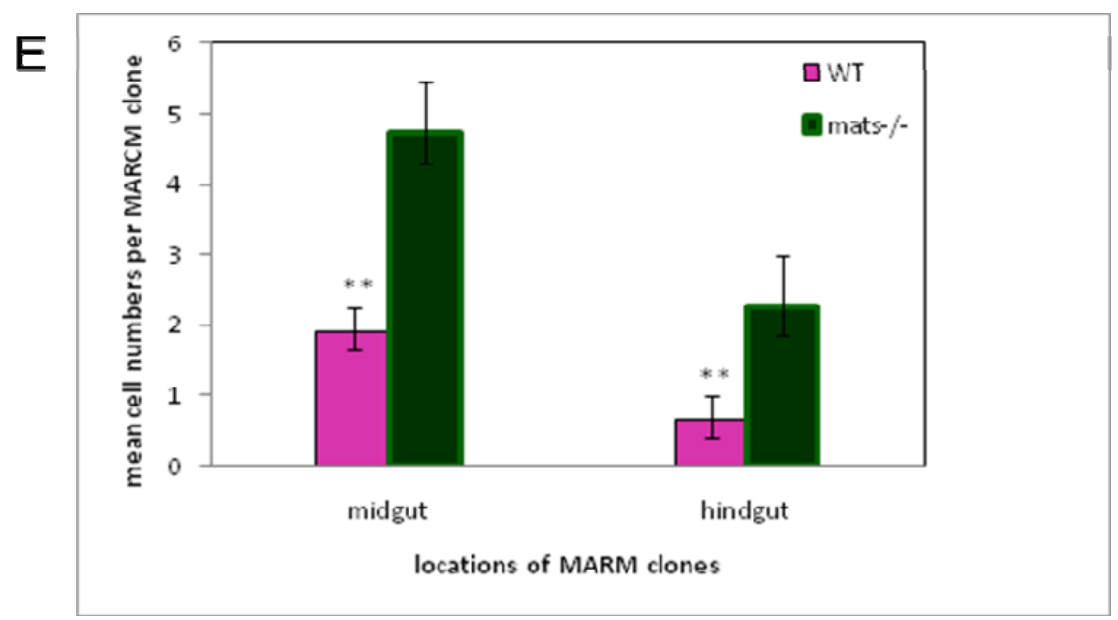

Figure 2. Loss-of-function of dmats-/- in stem cells of midgut enhanced their proliferation comparing to wild type stem cells

The strains ( w; hsFlp, UAS-GFP; FRT82B, Tub-GAL4, Tub-Gal80/96E or w; hsFlp, UAS-GFP; FRT82B, Tub-GAL4, Tub-Gal80/pi-myc or w; hsFlp, UAS-GFP; FRT82B, Tub-GAL4, Tub-Gal80/mats ${ }^{\text {roo }}$ ) were generated by crossing w; hsFlp, UAS-GFP; FRT82B, Tub-GAL4, Tub-Gal80/TM6B with w; FRT82B mats ${ }^{\text {roo }} /$ TM6B; w, FRT82B mats ${ }^{e 235} / T M 6 B, y, w ; P[r y+$; hsneo]; FRT82B P $[y+r y+] 96 E$ A or $y, w$,hsflp; FRT82B, hs pi-Myc. GFP labeled MARCM cell clones in midgut were observed and pictures were taken by Flowview1000 confocal microscope. The GFP signals were in cytosole, and the nuclei were shown as dark areas surrounded by the cytosol GFP. A and A', wild type GFP cell clones 100x in midgut after heat shock 6 days. C and C', wild type GFP cell clones after heat shock 6 days in higher magnification 400x. B and B', GFP labeled dmats-/- cell clones in midgut, after heat shock 6 days. D and D', GFP labeled dmats-/- cell clones after heat shock 6 days in higher magnification in midgut. E, after heat shock treatment, the cell numbers per MARCM clones in mdgut and hindgut were counted. Data were analyzed and students' T-test was performed. Figure showed the $8^{\text {th }}$ day of the clone size in midgut and hindgut. Error bars were standard error. Sample size of wild type MARCM clones in midgut was 12; sample size of mats-/- clones in midgut was 18; sample size of wild type clone in hindgut was 12; sample size of mats-/- clones in hindgut was $15{ }^{* *}$, indicating $\mathrm{p}<0.01$. 


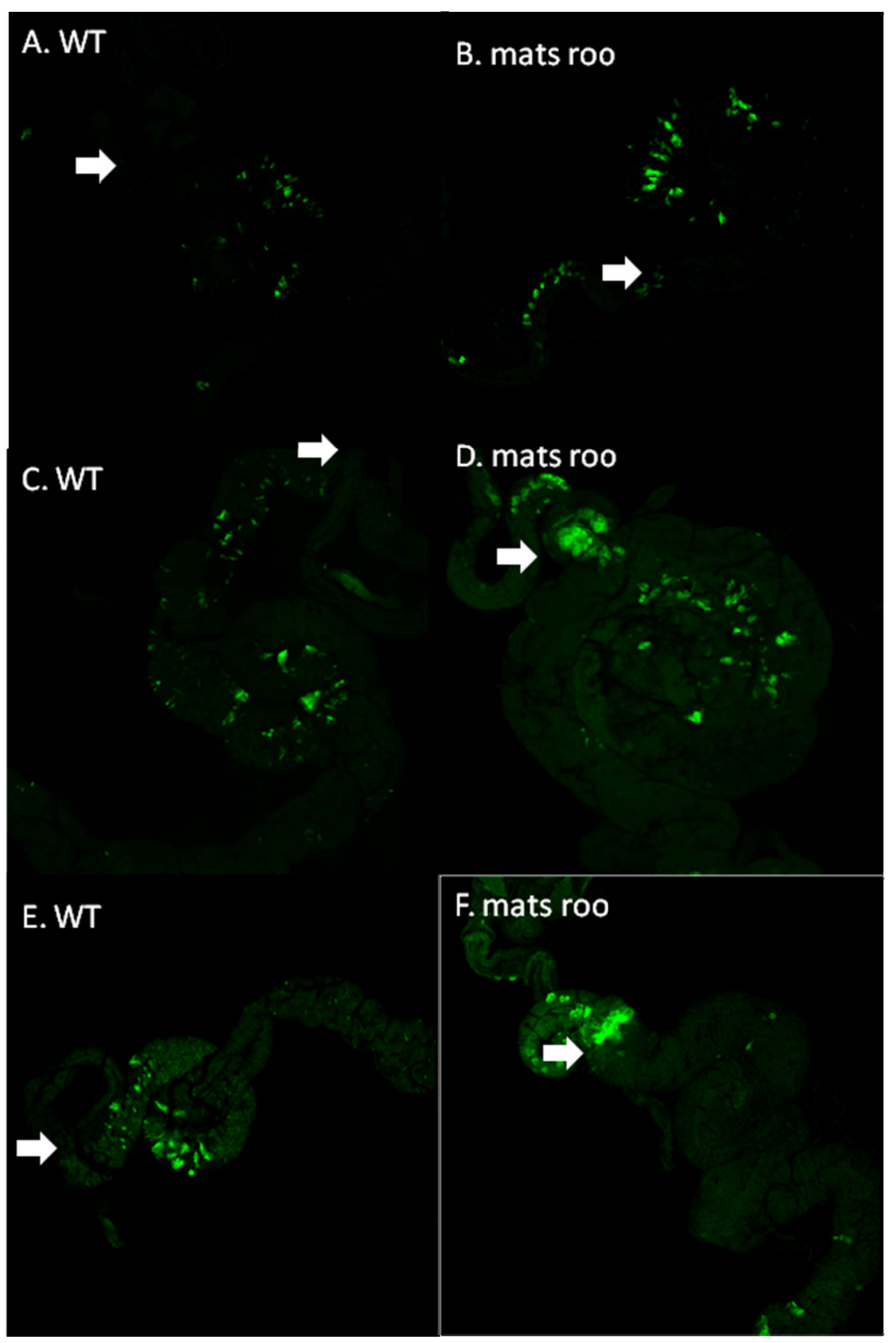

Figure 3. Chronic mutation of dmats-/- in stem cells of midgut caused tissue outgrowth

Fly strains using are the same as in Fig. 3. After heat shock, strains were maintained until the indicated time for gut phenotype observation. A, C, E ( w; hsFlp, UAS-GFP; FRT82B, Tub-GAL4, Tub-Gal80/96E) showed the wild type cell clones labeled by eGFP. B, D, E ( $w$; hsFlp, UAS-GFP; FRT82B, Tub-GAL4, Tub-Gal80/mats ${ }^{\text {roo }}$ ) showed the loss-of-function of dmats -/- mutant clones labeled by eGFP and showed bigger cell numbers in the clones. A and B samples were collected after 8 days heat shock treatment. C and D samples were harvested at the $15^{\text {th }}$ day after heat shock treatment. E and $\mathrm{F}$ samples were obtained at the $35^{\text {th }}$ day after heat shock treatment. The white arrow indicated the structure where overgrowth may occur. A'-E' showed the hindgut morphology of the corresponding samples of A-E. 

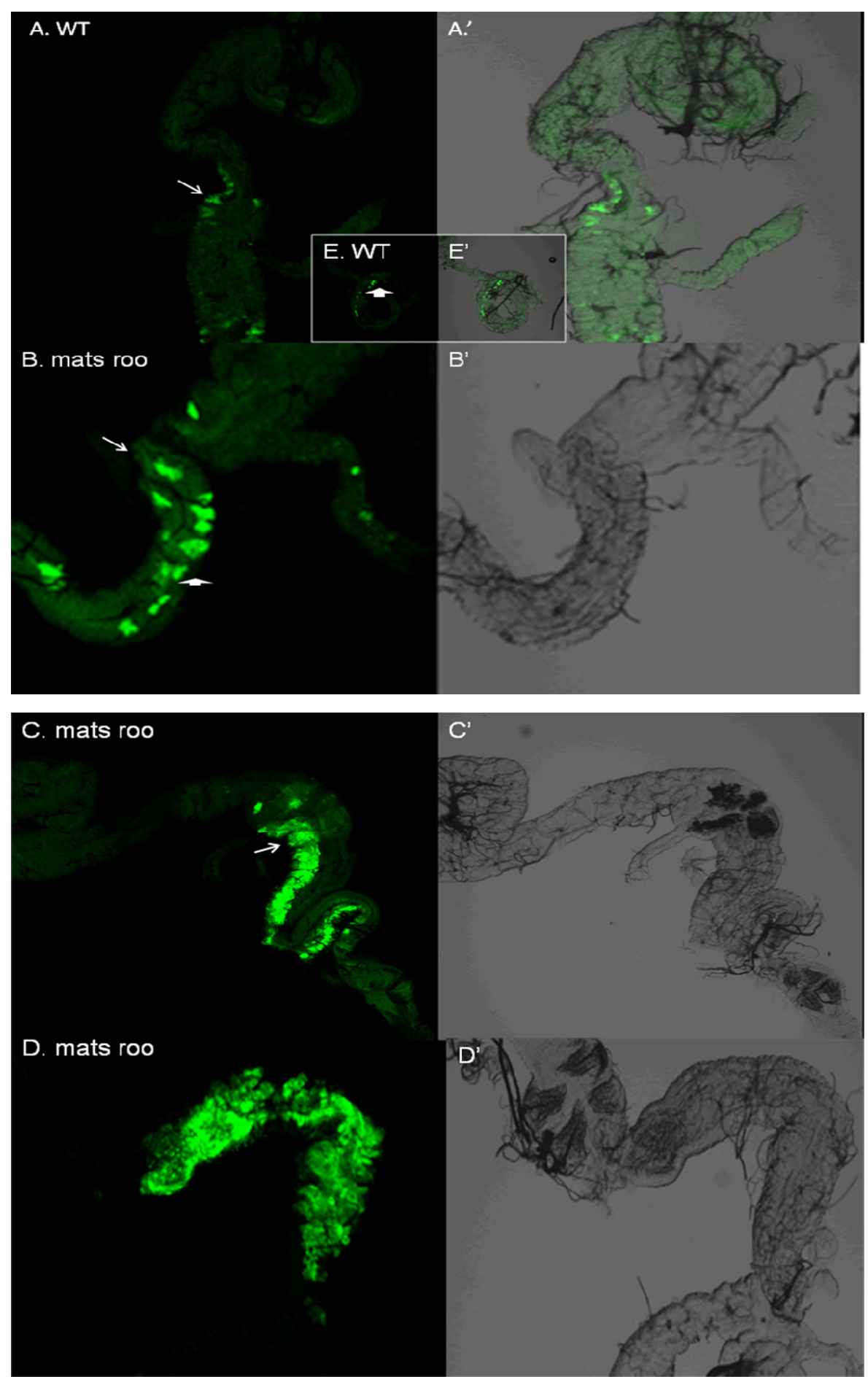

Figure 4. Loss-of-function of dmats-/- in stem cells of hindgut resulted in the bigger cell clones and tissue outgrowth than wild type

Fly strains that were used were the same as in Fig. 3. The gut phenotypes were observed by confocal microscope. A, A', E, E', the wild type GFP labeled cell clones in the hindgut 200x ( $w$; hsFlp, UAS-GFP; FRT82B, Tub-GAL4, Tub-Gal80/96E). B, B', C, C', D, D', GFP labeled dmats-/- mutant clones in the hindgut ( w; hsFlp, UAS-GFP; FRT82B, Tub-GAL4, Tub-Gal80/mats ${ }^{\text {roo }}$ ), 200x. D and D' showed the tissue outgrowth. The arrow indicated the stem cell clones. The arrow head showed the transient clones. After heat shock treatment, the fly samples were harvested at 6 th day or $35^{\text {th }}$ day: A-D samples were harvested on $35^{\text {th }}$ day, while E and E' was harvested on the $6^{\text {th }}$ day. 


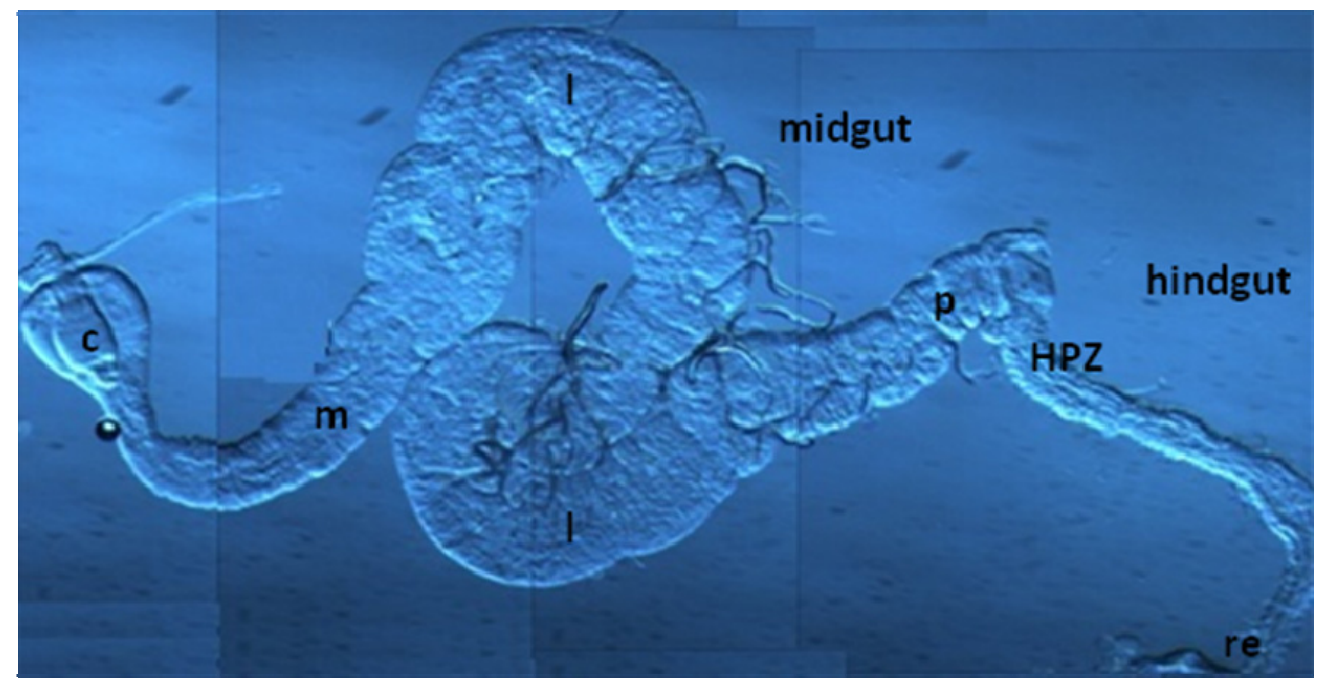

Sup 1. The dMats distribution in the midgut and hindgut of adult fruit flies.

A, The picture showed the structure of midgut and hindgut of adult Drosophila (100x). The whole gut of the fruit fly was dissected according to the protocol. The tissue was fixed and mounted on the glass slide. The structure is indicated as labeling. Midgut is from cardia to pylorus where the malpighian tubules reside and hindgut is from pylorus to the rectum. C: cardia; M: megan; 1 : 1 st loop and $2^{\text {nd }}$ loop; p: pylorus; HPZ: hindgut proliferation zone; re: rectum.

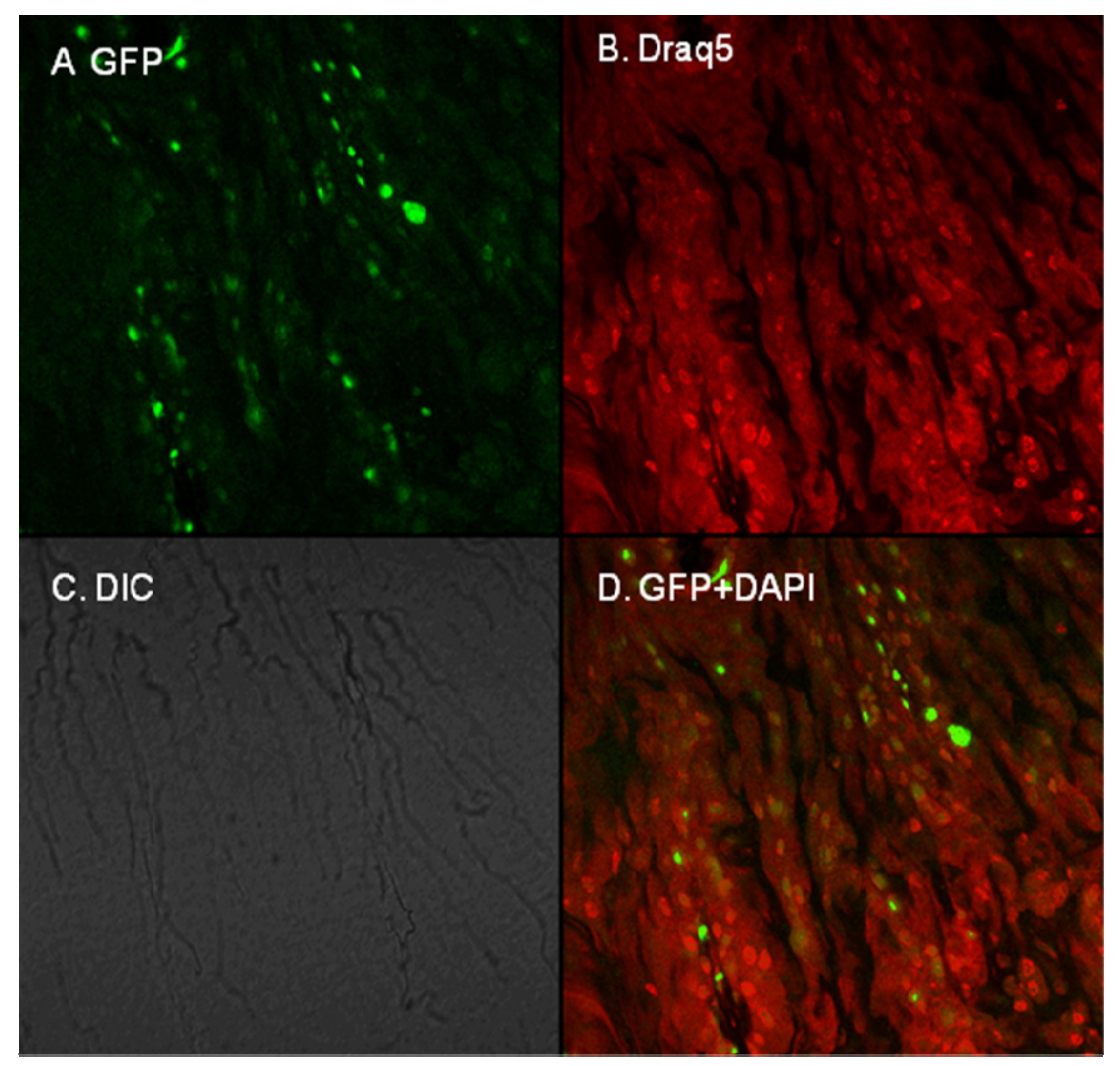

Sup 2. dMats signals in the midgut with nuclear stain by Draq5

Guts were obtained from fly strain containing the dmats driving eGFP signals were stained with nuclear dye Draq5. The eGFP signals showed the dMats expression in the cells of midgut. A, the GFP signals showed the dMats expression in the midgut cells. B, the red Draq5 signals stained the nuclear of cells. C, the view showed the light view of the midgut tissue. D, the merge view showed the GFP signals and nuclear signals, indicating dMats distribution in the diploid cells of midgut. 


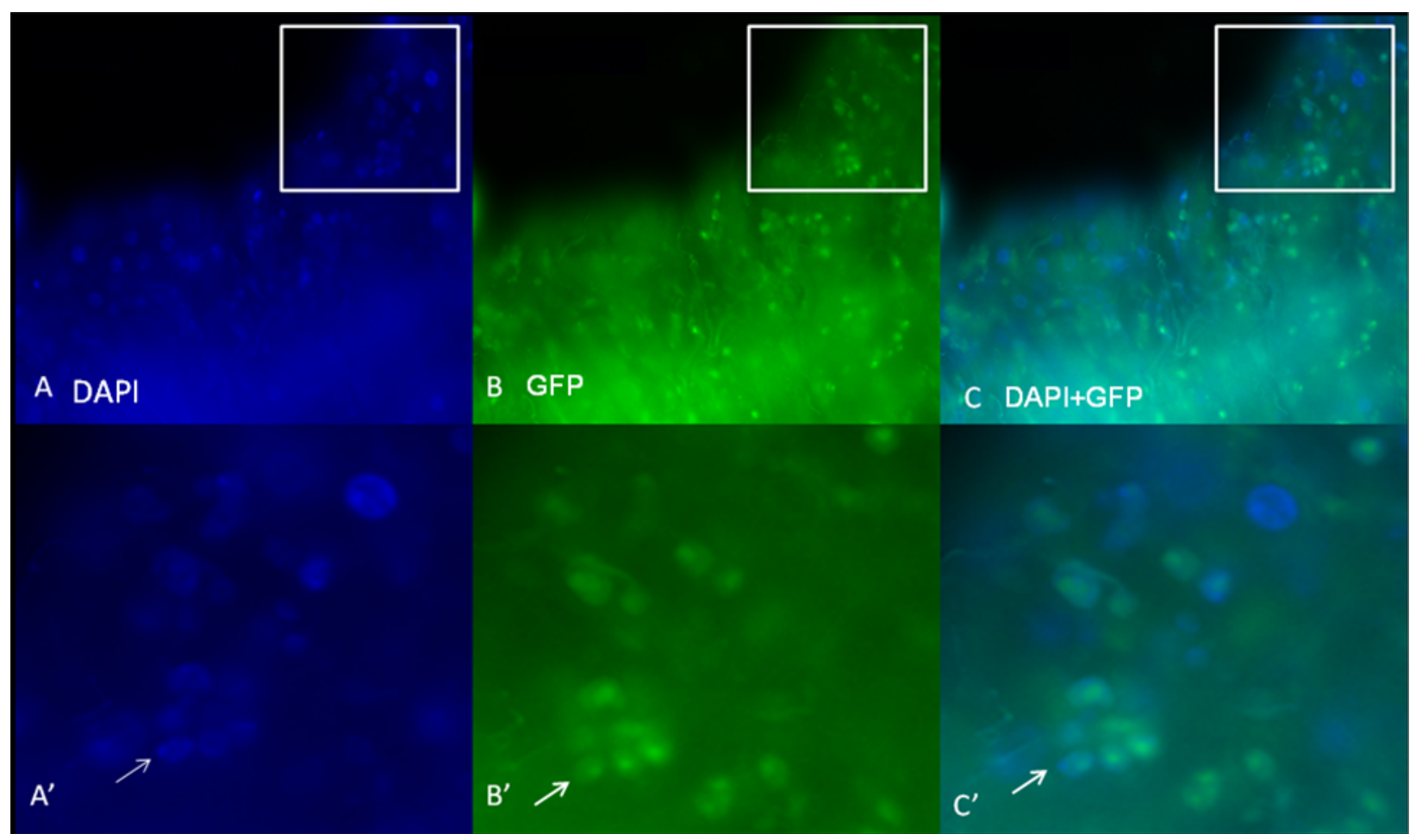

Sup 3. The dMats expressed in the population of diploid cells

The guts expressing dMats with GFP labeling were stained DAPI. A and A', the GFP signals indicated dMats expression in the cells. B and B', the DAPI signals showed the cell population in midgut. C and C', the merged view showed the dMats did not expressed in the polyploidy cells. A', B', C' are higher magnification of portions of the corresponding views. The arrow indicated ISC cell and its daughter cells. 